\title{
Upper-Mantle Shear Velocities beneath Southern California Determined from Long-Period Surface Waves
}

\author{
by J. Polet and H. Kanamori
}

\begin{abstract}
We used long-period surface waves from teleseismic earthquakes recorded by the TERRAscope network to determine phase velocity dispersion of Rayleigh waves up to periods of about $170 \mathrm{sec}$ and of Love waves up to about $150 \mathrm{sec}$. This enabled us to investigate the upper-mantle velocity structure beneath southern California to a depth of about $250 \mathrm{~km}$. Ten and five earthquakes were used for Rayleigh and Love waves, respectively. The observed surface-wave dispersion shows a clear Love/Rayleigh-wave discrepancy that cannot be accounted for by a simple isotropic velocity model with smooth variations of velocity with depth. Separate isotropic inversions for Love- and Rayleigh-wave data yield velocity models that show up to $10 \%$ anisotropy (transverse isotropy). However, tests with synthetic Love waves suggest that the relatively high Love-wave phase velocity could be at least partly due to interference of higher-mode Love waves with the fundamental mode. Even after this interference effect is removed, about $4 \%$ anisotropy remains in the top $250 \mathrm{~km}$ of the mantle. This anisotropy could be due to intrinsic anisotropy of olivine crystals or due to a laminated structure with alternating high- and low-velocity layers. Other possibilities include the following: upper-mantle heterogeneity in southern California (such as the Transverse Range anomaly) may affect Love- and Rayleigh-wave velocities differently so that it yields the apparent anisotropy; higher-mode Love-wave interference has a stronger effect than suggested by our numerical experiments using model 1066A. If the high Love-wave velocity is due to causes other than anisotropy, the Rayleigh-wave velocity model would represent the southern California upper-mantle velocity structure. The shear velocity in the upper mantle (Moho to $250 \mathrm{~km}$ ) of this structure is, on average, 3 to $4 \%$ slower than that of the TNA model determined for western North America.
\end{abstract}

\section{Introduction}

The seismic velocities in the upper mantle beneath the western United States are generally considered to be lower than average (e.g., the TNA model of Grand and Helmberger, 1984). In a broad sense, southern California is categorized as "western United States," but it is unclear whether its upper-mantle structure is indeed similar to that represented by, for instance, the TNA model. There are in fact different lines of evidence that suggest that the southern California upper mantle has significant structural heterogeneities. Large-scale mantle high-velocity zones such as the Transverse Ranges anomaly (Raikes, 1978; Hadley, 1978; Hadley and Kanamori, 1979; Humphreys and Clayton, 1990) and the Isabella anomaly (Aki, 1982; Jones and Kanamori, 1994) have been found.

To investigate the upper-mantle structure beneath southern California further, it is necessary to determine the structure to a relatively large depth $(200 \mathrm{~km})$ on the spatial scale of southern California. A useful approach to this problem is the surface-wave phase velocity method. The dispersion of surface waves has been widely used to determine seismic velocity structures in the Earth's crust and mantle. In previous studies in southern California, Press (1956) and Ewing and Press (1959) used Rayleigh waves to determine crustal structures. Brune and Dorman (1963) used both Rayleigh and Love waves to study the crust and upper-mantle structure beneath the Canadian shield, and McEvilly (1964) determined a crust-upper-mantle structure for the central United States by inverting Rayleigh- and Love-wave dispersion curves.

Due to the limited quality of the data, the earlier measurements of surface-wave phase velocities in southern California were limited to periods shorter than $27 \mathrm{sec}$. Recently, Wang and Teng (1994) determined Rayleigh-wave phase velocities in southern California to a period of $100 \mathrm{sec}$ using broadband data obtained from TERRAscope. Stange and Friederich (1993) determined the dispersion of fundamental- 
mode Rayleigh waves in southern Germany in the frequency range 4 to $50 \mathrm{mHz}$ from data recorded at a broadband network. The purpose of the present study is to provide new constraints on the average velocity structure of southern California by determining surface-wave phase velocities for periods up to $170 \mathrm{sec}$. This increased period range is critically important for exploring deeper structures. The main objective is to determine the overall velocity structure in the top $250 \mathrm{~km}$ of the upper mantle for both Rayleigh and Love waves. In view of the limited resolution of surface-wave methods, we do not intend to resolve the details of the vertical variation of velocities nor do we attempt a tomographic inversion for the lateral variations within the network.

\section{Method}

In this study, we use a traditional method to determine the phase velocities (e.g., Press, 1956; Aki, 1961), which we will describe briefly. First the phase travel times are calculated as a function of period. This is done by computing the Fourier transform of the surface wave train $x(t)$ for each station $i$ :

$$
\hat{x}_{i}(\omega)=\int_{-\infty}^{+\infty} x_{i}(t) \cdot e^{-i \omega t} d t
$$

where $\omega$ is the angular frequency.
The phase $\psi_{i}(\omega)$ of the signal is then determined by

$$
\psi_{i}(\omega)=\arctan \frac{\operatorname{Im}\left[\hat{x}_{i}(\omega)\right]}{\operatorname{Re}\left[\hat{x}_{i}(\omega)\right]} .
$$

The phase travel time $t_{i}(\omega)$ can consequently be calculated using

$$
t_{i}(\omega)=-\frac{\psi_{i}(\omega)-\omega \cdot\left(t_{s i}-t_{0}\right)+2 \pi N}{\omega}
$$

where $t_{s i}$ is the starting time of the surface-wave signal, $t_{0}$ is the origin time of the event, and $N$ is an integer that can be determined unambiguously from the approximate phase velocity known for the area. When the $t_{i}$ 's for all stations are evaluated, the phase velocity $C(\omega)$ and the propagation azimuth $\Phi(\omega)$ within the network can be determined from the following set of equations in the least-squares approximation, with the main assumption that we are dealing with a plane wave within the network.

$$
\begin{aligned}
\Delta_{i} \cdot \cos \phi_{i} \cdot \frac{\cos \Phi(\omega)}{C(\omega)}+\Delta_{i} \cdot \sin \phi_{i} \cdot \frac{\sin \Phi(\omega)}{C(\omega)} \\
=t_{i}-t_{r}\left(\text { for } i=1, \ldots, N_{s}-1\right)
\end{aligned}
$$

where $\Delta_{i}$ is distance of station $i$ measured from a reference

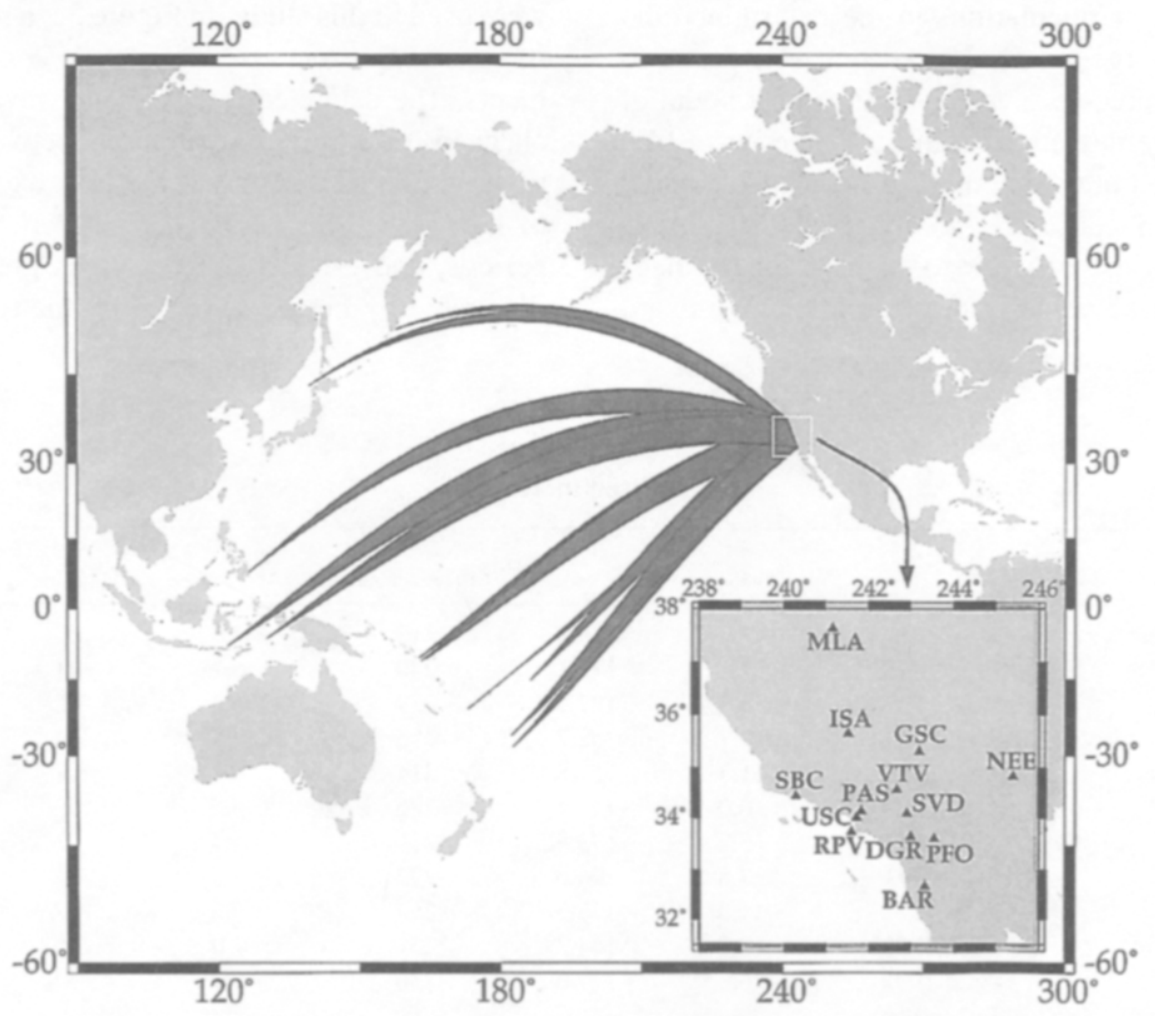

Figure 1. Great circle paths of the events used in this study. Insert shows location of TERRAscope stations in southern California; exact locations can be found on wwwpage http://www.gps.caltech.edu/terrascope/TerraInfo.html. 
station $r, \phi_{i}$ is azimuth of station $i$ measured from station $r$, $t_{r}$ is phase arrival time at reference station, and $N_{s}$ is number of stations.

\section{Data Selection}

The data we used for this study are long-period velocity records provided by the TERRAscope network located in southern California. The locations of the stations in the network are given in Figure 1 (insert). Out of the 13 stations, PAS, GSC, PFO, SBC, ISA, and BAR use the Streckeisen STS1 seismometer and the stations SVD, USC, MLA, VTV, NEE, RPV, and DGR use the Streckeisen STS-2. We equalized the response of STS-2 to STS-1 so that accurate phase velocity measurements could be made. BAR, VTV, NEE, RPV, and DGR became operational during the course of this study, so their data were not available for all the events studied. For Rayleigh waves, we used the vertical components of the records. The transverse components obtained by rotation of the horizontal records according to the source-receiver azimuth were used for the Love-wave analysis.

About 15 large events with long oceanic paths to the TERRAscope network were analyzed. Only shallow events were used to minimize contamination of the fundamental mode with higher-mode surface waves. We determined Rayleigh- and Love-wave phase velocities for ten and five of these events (Fig. 1 and Table 1), respectively. We show a representative example of these data in Figure 2. Data were rejected if the errors or fluctuations in the determined dispersion curves were large. Two main reasons were found for these problems: a source-receiver azimuth near a nodal direction and/or a large deviation from the plane wave-front approximation as used in this method. A near-nodal sourcereceiver azimuth can cause the source phase of surface waves to vary across the network to such an extent that it impedes accurate phase velocity determination. We exam- ined the shape of the wave front (for different frequencies) by first calculating the distance between the stations parallel to the direction of the ray as calculated in equation (1). Subsequently, we performed a linear regression on these points as a function of differential phase arrival time. The fit of these points on a straight line, as indicated by the correlation coefficient, determines the deviation of the wave front from a plane. Over $95 \%$ of the Rayleigh-wave phase measurements used in this study were determined to have correlation coefficients greater than 0.9975 (a correlation coefficient of 1 denoting perfect fit on a straight line). For the Love-wave measurements, over $90 \%$ of the measurements used in the inversion had a correlation coefficient greater than 0.9950 . Figure 3 shows typical examples for the event near the Vanuatu Islands [Table 1, (a) and (b)] and the event near Tonga in (c). In Figure 3a, the Love-wave measurements show a strong deviation from a plane wave front for this event, with a correlation coefficient of only 0.8883 . This could be due to the fact that Love waves are more sensitive to near-surface structures (shallow lateral inhomogeneities) and probably suffer more from refraction effects from the continental margin. These data clearly show an unacceptable deviation from the plane-wave approximation (which is also obvious in the large standard error of the measured phase velocities), and thus the resulting dispersion curve was not used in our inversion. The Love-wave data retained for the inversion do not have this problem. Figure $3 b$ shows one of the worst fits on a plane wave for the Rayleigh-wave measurements that were used in this study. In Figure 3c, a more typical example is given, representative of over $95 \%$ of the used measurements. The data clearly fit a plane wave very well. We also checked for amplitude variations between the different stations and found that they very rarely exceed $10 \%$. Coherence of the waveforms across the network is very good for all periods, and especially for periods greater than $50 \mathrm{sec}$, as illustrated by Figure 4 . Based on the results of these inves-

Table 1

Events Used in This Study

\begin{tabular}{|c|c|c|c|c|c|c|c|c|}
\hline Location & Date & Latitude & Longitude & Backazimuth & Magnitude & $\begin{array}{l}\text { No. } \\
\text { Stations }\end{array}$ & Love & Rayleigh \\
\hline Sea of Japan & 7 July 1993 & 43.2 & 139.4 & 312 & 7.6 & 5 & & $\mathrm{X}$ \\
\hline Vanuata Islands & 30 June 1993 & -21.1 & 173.1 & 240 & 6.7 & 10 & & $\mathrm{X}$ \\
\hline Kermadec Islands & 18 June 1993 & -28.4 & -177.2 & 239 & 6.7 & 11 & & $\mathrm{X}$ \\
\hline Solomon Islands & 12 June 1993 & -10.7 & 162.7 & 255 & 6.1 & 6 & & $\mathrm{X}$ \\
\hline East coast of Kamchatka & 8 June 1993 & 51.3 & 157.8 & 314 & 7.1 & 8,7 & $\mathrm{X}$ & \\
\hline Mariana & 6 June 1993 & 6.0 & 146.4 & 286 & 6.5 & 9 & & $\mathrm{X}$ \\
\hline Tonga Islands & 16 May 1993 & -15.2 & -173.5 & 236 & 6.7 & 10 & & $\mathrm{X}$ \\
\hline Mindanao, Philippines & 11 May 1993 & 7.8 & 126.6 & 292 & 6.6 & 3 & $\mathrm{X}$ & \\
\hline Solomon Islands & 6 March 1993 & -11.0 & 163.5 & 254 & 6.6 & 6,7 & $\mathrm{X}$ & $\mathrm{X}$ \\
\hline Santa Cruz Islands & 6 March 1993 & -10.9 & 164.2 & 254 & 7.0 & 6,6 & $\mathrm{X}$ & $\mathrm{X}$ \\
\hline South of Fiji Islands & $\begin{array}{l}6 \text { March } 1993 \\
20 \text { December }\end{array}$ & -26.3 & -177.6 & 230 & 6.7 & 5 & & $\mathrm{X}$ \\
\hline Banda Sea & $\begin{array}{c}1992 \\
12 \text { December }\end{array}$ & -6.5 & 130.3 & 277 & 7.1 & 5 & $\mathrm{X}$ & \\
\hline Flores Indonesia & 1992 & -8.2 & 121.9 & 280 & 7.5 & 5 & & $\mathrm{X}$ \\
\hline
\end{tabular}


Santa Cruz Islands, $\Delta=86^{\circ}$, back-azimuth $=254^{\circ}$, magnitude $=7.0$

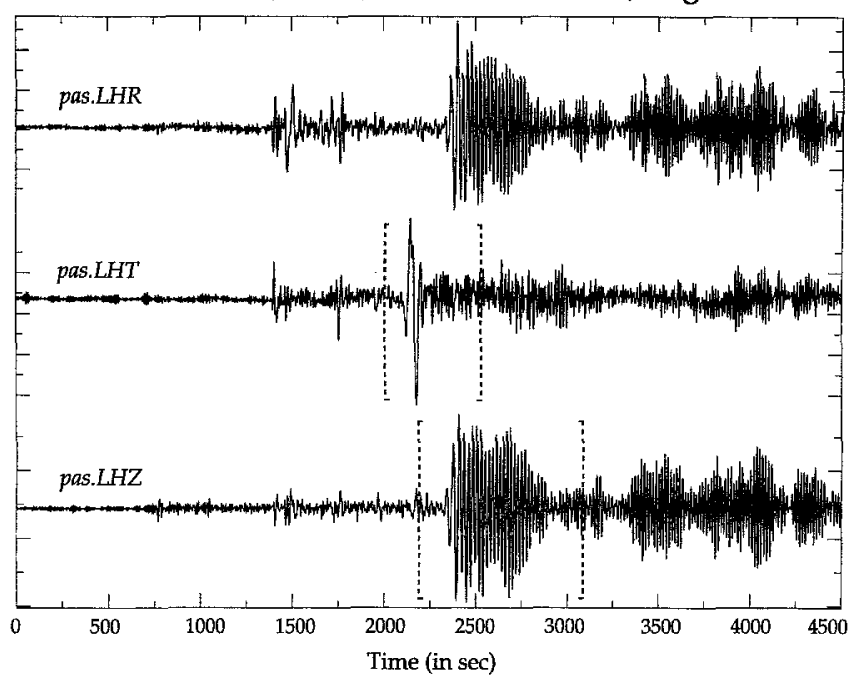

Figure 2. Long-period velocity records from station PAS for the event near the Santa Cruz Islands (Table 1). Vertical lines indicate time windows used in the determination of phase velocity dispersion.

tigations, we believe that accurate determination of phase velocities in this region is possible, even though lateral heterogeneities may exist.

\section{Data Analysis: Phase Velocity Measurements}

The phase velocity values we determined are shown in Figure 5. The standard error is about $\pm 0.07 \mathrm{~km} / \mathrm{sec}$ for most data points. Love-wave velocities for periods longer than $150 \mathrm{sec}$ and shorter than $50 \mathrm{sec}$ exhibit large scatter and are questionable. Rayleigh-wave velocities are generally well determined for periods between 20 and $180 \mathrm{sec}$, although they usually have larger errors for periods shorter than 50 sec. The dispersion curves for the individual events display some irregular fluctuations as a function of period. This is probably due to interference of waves caused by the lateral heterogeneities between the source and the TERRAscope network as well as within the network, since the fluctuations of the dispersion curves generally coincide with (albeit small) deviations in the great circle direction (rarely exceeding $5^{\circ}$ ) and smaller correlation coefficient $r$ for the fit to a plane wave of the phase measurements. This pattern of fluctuation varies for different events, but the average dispersion curves obtained from the different events are overall in good agreement (generally falling within one standard error of each other), and when the results from all the events are averaged, a smooth dispersion curve was obtained (Fig. 6), showing that the effects of these lateral heterogeneities are probably averaged out. Since our objective here is to determine the gross structure, rather than the details, in Figure 6, we first compare the observed dispersion curves with those of several standard continental models to demonstrate the overall differences. The models used here are the Gutenberg model
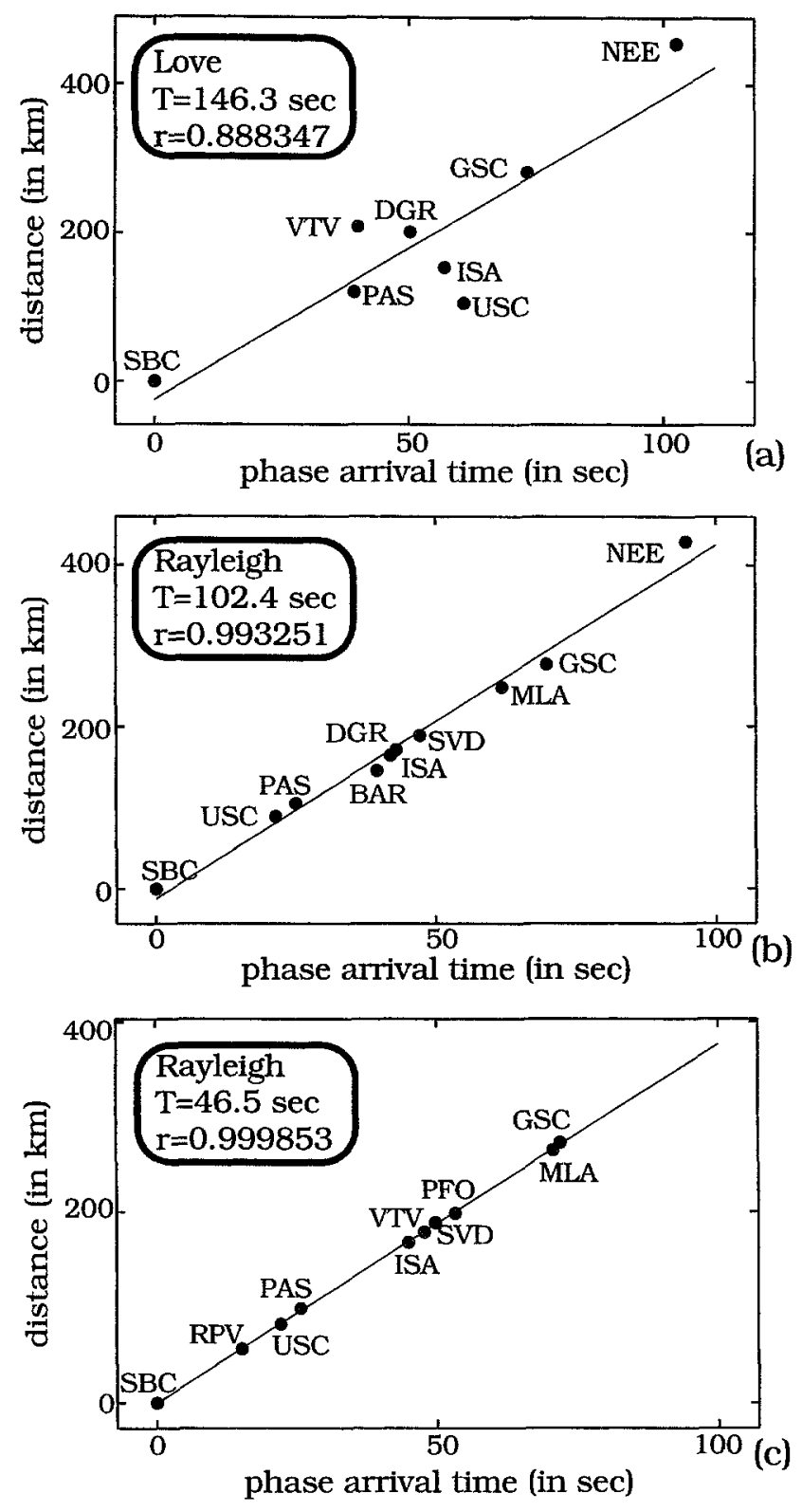

Figure 3. (a) Distance along ray direction versus phase arrival time for Love-wave data from an event near the Vanuatu Islands (Table 1) for a period of $146.3 \mathrm{sec}$. The cross-correlation coefficient $r$ is 0.8883. (b) Same for Rayleigh-wave data for a period of $102.4 \mathrm{sec}$. Value of $r$ is 0.9932 . (c) Same for Rayleigh-wave data for a period of $46.5 \mathrm{sec}$ for the event near Tonga. Value of $r$ is 0.9999.

(continent), the JB model (continent) (parameters for both the Gutenberg as well as the JB model were obtained from Dorman et al., 1960), the SNA model (shield, Grand and Helmberger, 1984), and the TNA model (western North America, Grand and Helmberger, 1984). The Rayleigh-wave velocities are lower than those calculated for the Gutenberg model, the JB model, and the SNA model. They are also significantly lower than the phase velocities predicted by the TNA velocity model, with a low-velocity crustal model used for the top $32 \mathrm{~km}$. 


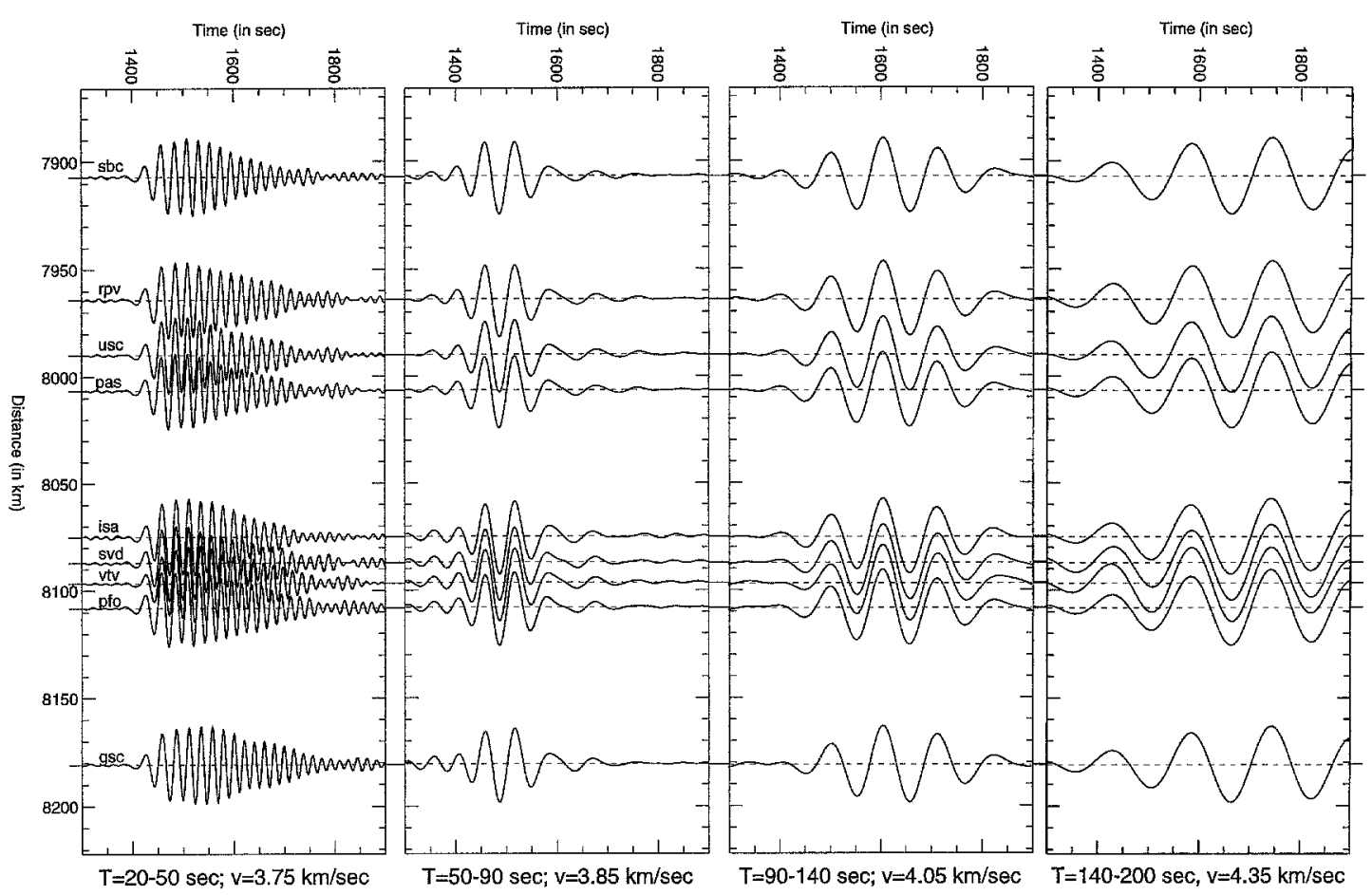

Figure 4. Reduced record sections for bandpass-filtered seismograms.

The Love-wave phase velocity measurements have slightly larger errors but also are in good agreement for the different events. In contrast to the Rayleigh waves, the average values of Love-wave phase velocities are clearly higher than those predicted by the TNA model (Fig. 6b) and are in the same range as those for the standard continental models like the Gutenberg and JB models.

For one of the events (located in the Santa Cruz Islands), we could determine the phase velocity using the G2 wave, coming from the opposite azimuth. The result is very similar to that obtained from the $\mathrm{G} 1$ data for a period range of 80 to $150 \mathrm{sec}$ (for smaller periods, the G2 wave contained substantial non-plane-wave energy).

\section{Inversion Method}

Since, as mentioned earlier, our primary interest here is in the average velocity structure, we use the simple leastsquares inversion method by Takeuchi et al. (1964), with the emphasis on obtaining smooth structures. We briefly describe the method in the following.

In essence, a reference structure (here the Gutenberg model) is chosen first. Then this model is perturbed until it can explain the observed dispersion curves satisfactorily. The perturbations are determined from the difference between the observed phase velocities and those computed for the reference structure using partial derivatives of phase velocity with respect to the $S$-wave velocity in each layer. We do not perturb density and $P$-wave velocity since the partial derivatives of phase velocity with respect to these parame- ters are relatively small. Then we solve for the following set of equations (one for each period for which phase velocity measurements have been made):

$$
\Delta C=\sum_{i} \Delta \beta\left(r_{i}\right) \cdot\left(\frac{\partial C}{\partial \beta}\right)_{a, \rho}\left(r_{i}\right)\left(\text { for } i=1, \ldots, N_{l}\right)
$$

where $\Delta C$ is the difference in phase velocity at a given period from the reference model (here, Gutenberg model), $\Delta \beta\left(r_{i}\right)$ is the resulting difference in velocity in layer $i$ with the Gutenberg model, and $N_{l}$ is the number of layers.

\section{Inversion Results}

As shown in Figure 5, none of the existing models can explain the averages of the Love- and Rayleigh-wave phase velocity measurements simultaneously. In general, the observed Love-wave phase velocities tend to be faster than expected from the Rayleigh-wave velocities. As Babuska and Cara (1991) demonstrated, this is a fairly commonly observed trend for many places in the world.

An attempt to explain the Love- and Rayleigh-wave phase velocities (over a period range of 49 to 146 and 17 to $171 \mathrm{sec}$, respectively) simultaneously with an isotropic model leads to an unrealistic velocity model, as shown in Figure 7. Although we also used the shorter-period ( 20 to $50 \mathrm{sec}$ ), noisier, Rayleigh-wave phase velocities in this inversion, leaving them out would only have a negligible effect on the gross structure. This model exhibits strong fluctuations of velocity with depth even though the inversion was 

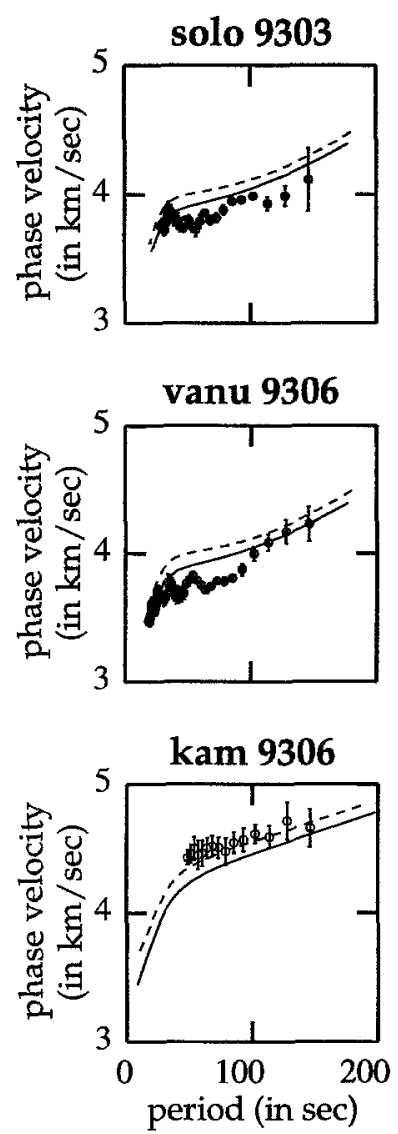

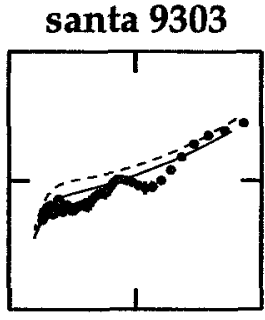

ker 9306

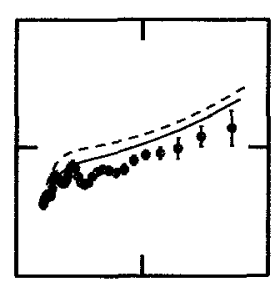

phil 9305

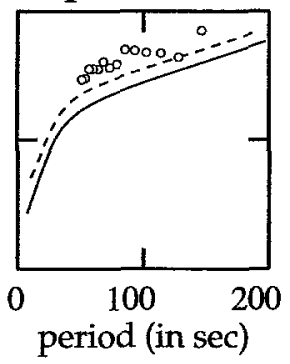

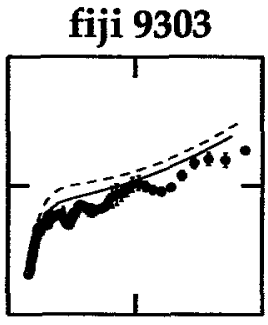
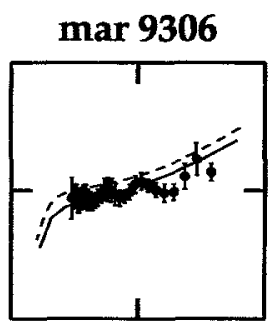

solo 9303

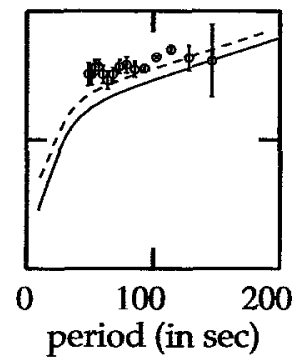

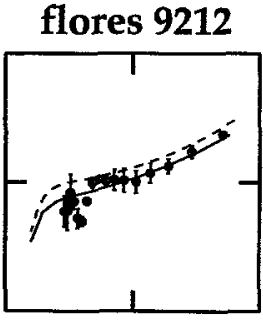

solo 9306

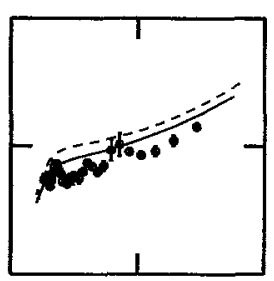

santa 9303

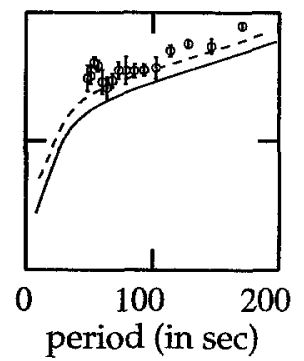

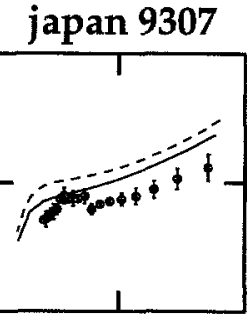

tonga 9305

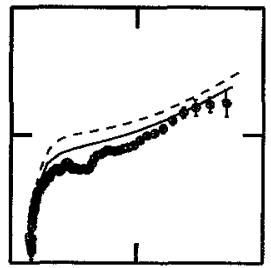

banda 9212

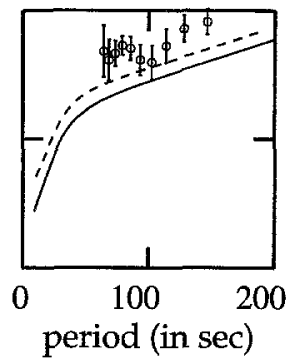

Figure 5. Determined phase velocity values. Open circles denote Love-wave values; dark circles, Rayleigh-wave values. Solid and dotted lines indicate reference dispersion curves calculated for the TNA and the Gutenberg models, respectively. Error bars show standard error.

damped. The relatively high velocities near the top of the mantle are a result of the greater sensitivity of Love-wave phase velocity for shallower layers, whereas the low-velocity zone at greater depths is required to explain the slower Rayleigh-wave velocity. This model is not realistic and still does not fit the measured Love-wave velocities well.

We then performed independent inversions of the observed Rayleigh- and Love-wave dispersion curves. The resulting velocity models ( $S V$ velocity for Rayleigh, $S H$ velocity for Love) are also shown in Figure 7 and the dispersion curves in Figure 8. The calculated phase velocity curves fit the data of both Rayleigh and Love waves well. Some damping was used in these inversions, to minimize the velocity fluctuations between adjoining layers and to minimize the velocity change for the lower layers, for which the resolution is low. The significance of the Love/Rayleighwave discrepancy is evident from the dashed and dotted dispersion curves in Figure 8, which indicate Love-wave phase velocities predicted from the Rayleigh-wave model and vice versa. The performed separate isotropic inversion is not strictly correct when dealing with an anisotropic structure (Mitchell, 1984; Kirkwood, 1978). However, for the period range of this study $\left(\partial C / \partial \beta_{S H}\right) \approx 0$ for Rayleigh waves and $\left(\partial C / \partial \beta_{S V}\right) \approx 0$ for Love waves (Figs. 3 and 12 of Anderson and Dziewonski, 1982), and the separate inversion would yield correct structures for $S H$ and $S V$, if only the $S$-wave velocity is anisotropic. In isotropic media, $P$-wave velocity has little effect on Rayleigh-wave phase velocity for the depth and period ranges concerned here. In anisotropic media, Rayleigh-wave phase velocity depends on the two partials with respect to vertical and horizontal $P$-wave velocity, $\left(\partial C / \partial \alpha_{P H}\right)$ and $\left(\partial C / \partial \alpha_{P V}\right)$. However, the $P$-wave partials are only in the range of about $20 \%$ of the $S$-wave partial (Fig. 12 of Anderson and Dziewonski, 1982), so that our conclusion on $S H / S V$ would not be significantly affected.

A difficulty in the determination of Love-wave phase velocities is the interference of higher modes with the fundamental mode (Thatcher and Brune, 1969). To avoid the contamination of the fundamental mode with higher-mode Love waves, we chose shallow events with long paths (Knopoff, 1983, 1972) to TERRAscope. To examine the possibility of higher-mode interference, we carried out synthetic tests. By mode summation, using the $1066 \mathrm{~A}$ velocity model (Gilbert and Dziewonski, 1975), we produced synthetic seismograms for those events that were used in our Love-wave phase velocity calculation. The results for two of these 

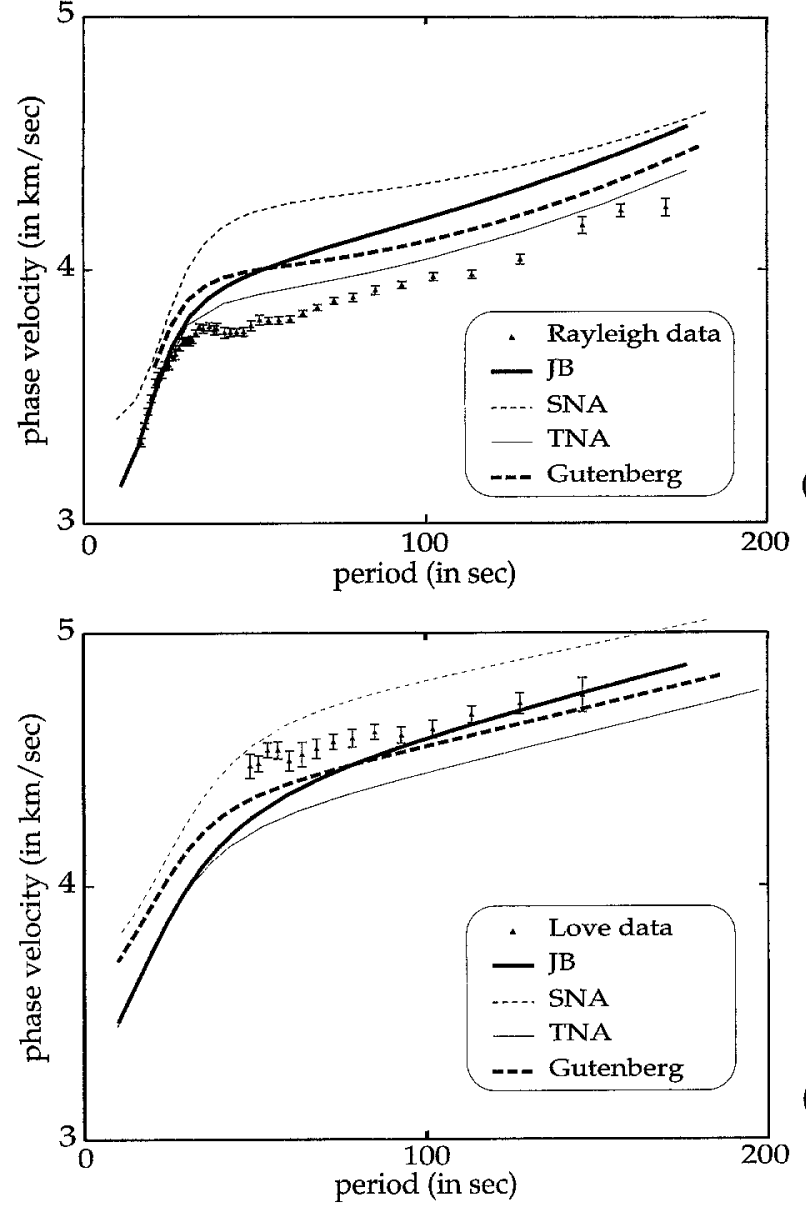

Figure 6. (a) Average Rayleigh-wave dispersion measurements and dispersion curves calculated for JB, SNA, TNA, and Gutenberg velocity models. (b) Same for Love-wave dispersion.

events are shown in Figures 9a and 9b; these are representative of all the events. As can be clearly seen in these synthetic seismograms, higher modes are present in the synthetics for Love waves in the same time window in which the fundamental mode is expected to arrive. For Rayleigh waves, however, the higher modes arrive outside of this time window. By comparison of these synthetic seismograms with the recorded seismogram (low passed at $0.0143 \mathrm{~Hz}$ or $70 \mathrm{sec}$ in period), we can see that the data contain higher modes. Synthetic seismograms like these were used to compute dispersion curves for the fundamental mode and for the total signal for all five of the events, using the same time windows as were used for the data. As expected, the resulting dispersion curves were the same for all the fundamentalmode seismograms. Because the epicentral distance is not the same for all the events, the interference effects also differ, so that the results for the seismograms that included the higher-mode Love waves were not identical but very similar. We subtracted the phase velocities determined for fundamental-mode synthetics from the average phase velocities obtained using total-mode summation and used these values

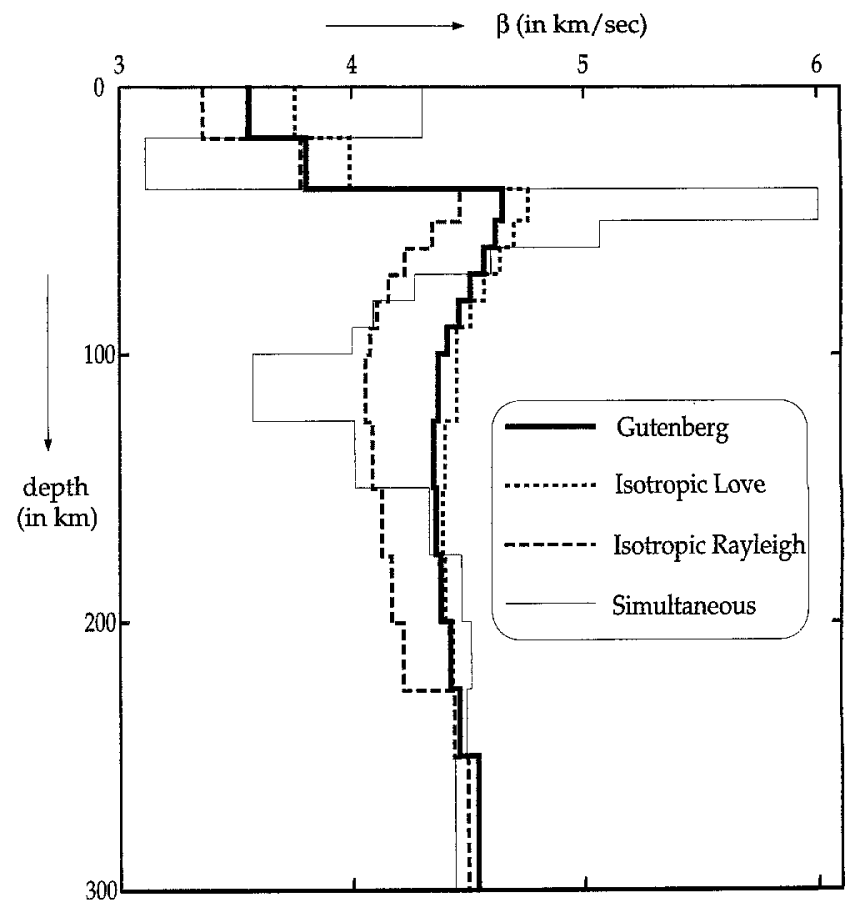

Figure 7. Shear-wave velocity-depth models. Thick solid line denotes Gutenberg model; thin solid line indicates the results of the simultaneous inversion of Rayleigh- and Love-wave phase velocities, and dotted lines show the results of the independent isotropic inversions.

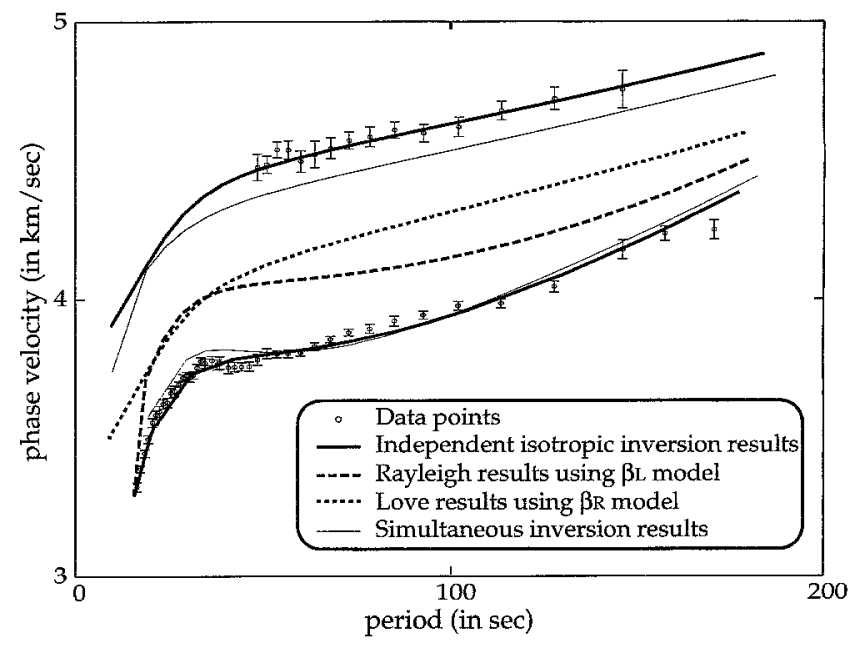

Figure 8. Observed (dot-bar symbols) and modeled (full and dotted lines) phase velocities for southern California. The error bars indicate the standard deviation of the measurements. The dotted lines show velocities predicted from the inappropriate model $\left(\beta_{L}\right.$ for Rayleigh waves and vice versa). The thick solid line indicates the results of the independent isotropic inversion, the thin solid line shows the results predicted by the simultaneous isotropic inversion. 


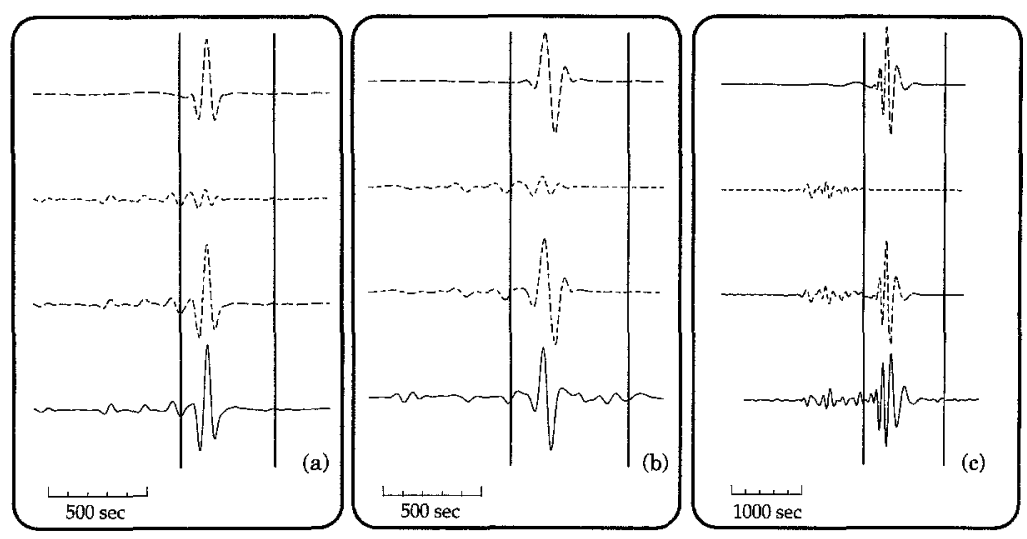

Figure 9. Synthetic and observed seismograms for station PAS low-pass filtered at 0.014 Hz. The top trace shows synthetics for fundamental mode only, the second trace shows the higher modes only, the third shows the total synthetic, and the bottom trace is the recorded data. (a) Love waves for the event in the Philippines (Table 1). (b) Love waves for the event near the Santa Cruz Islands. (c) Rayleigh waves for the same event. as a correction for our measured Love-wave dispersion results. Although the correction is dependent on the velocity model used, we think that this correction is reasonable, since the computed waveform synthetics agree well with the data, and we are looking at fairly long-period (greater than $60 \mathrm{sec}$ ) waves here. We repeated the inversion for the (now corrected) Love-wave data separately and simultaneously with the original Rayleigh-wave data, and the results are shown in Figures 10 and 11. Again, the velocity model resulting from the simultaneous inversion shows fluctuations and unrealistic velocities (Fig. 11), though to a lesser degree; also, the Love-wave velocity model $\left(\beta_{\mathrm{L} 2}\right)$ still does not fit the Rayleigh-wave data and vice versa (Fig. 10).

From these results, we conclude that if the correction found by these synthetic tests using the $1066 \mathrm{~A}$ velocity model is correct, around 4\% anisotropy is needed to explain the measured Rayleigh- and Love-wave dispersion and that the shear-wave velocity ( $S V$ ) beneath southern California determined from Rayleigh waves is significantly lower than that of the TNA model (Fig. 11). To confirm this last conclusion, we performed forward modeling and inverted the

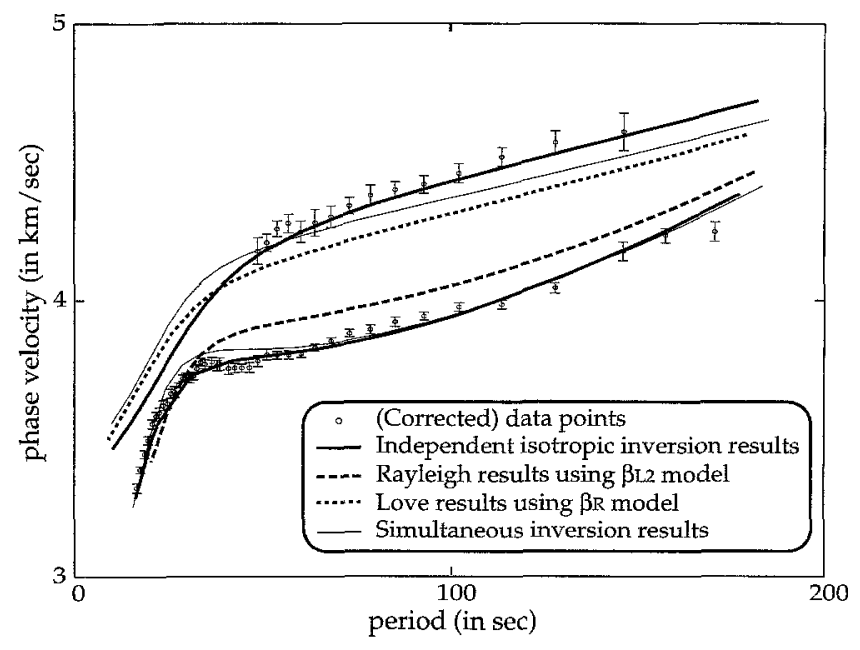

Figure 10. Same as Figure 8. Corrected Lovewave data are used. measured Rayleigh-wave dispersion data using the TNA model instead of the Gutenberg model as our starting model. In the forward modeling, we constructed three different velocity models from the TNA model by reducing the shear velocity by 2,4 , and $6 \%$ between Moho and $225 \mathrm{~km}$. These models are called TNA-2\%, TNA-4\%, and TNA-6\%, respectively (Fig. 12). Figure 13 shows the dispersion curves calculated using these models. It is evident that our data cannot be explained by TNA, or even the TNA-2\% model, but on average, the data lie somewhere between the values predicted by the TNA- $2 \%$ and TNA- $4 \%$ models.

We repeated our inversion using the Rayleigh-wave dispersion data, this time with TNA as our starting model. The resulting velocity model (Fig. 12) is very similar to that obtained using the Gutenberg model as our starting model, al-

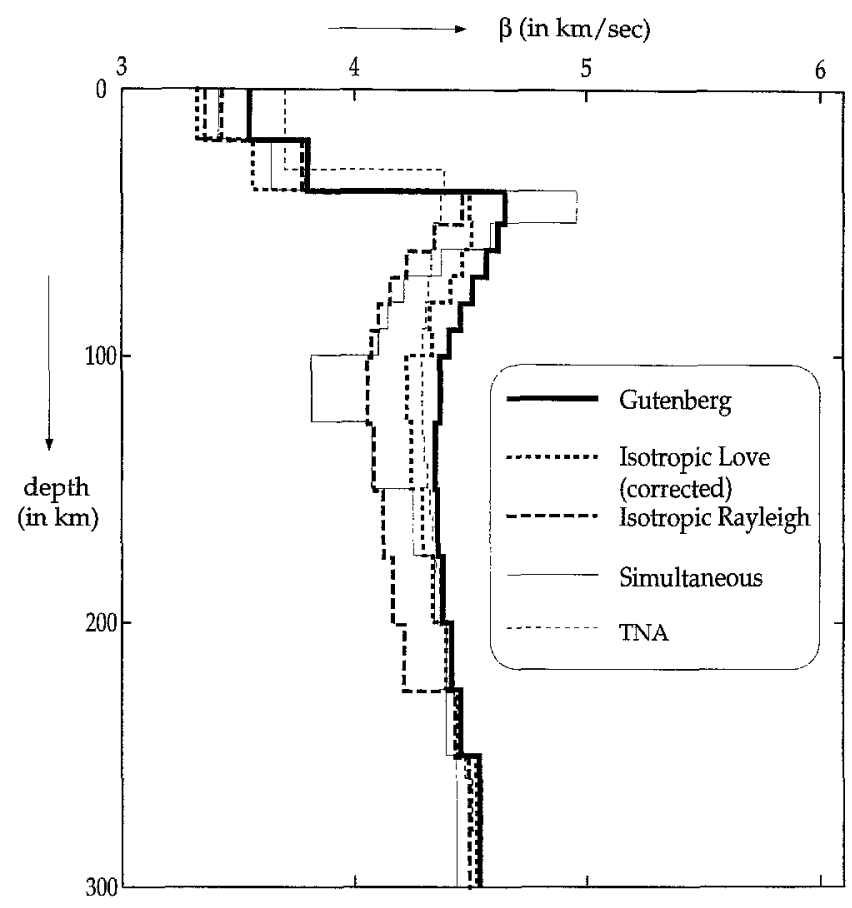

Figure 11. Same as Figure 7. Corrected Lovewave data are used. 


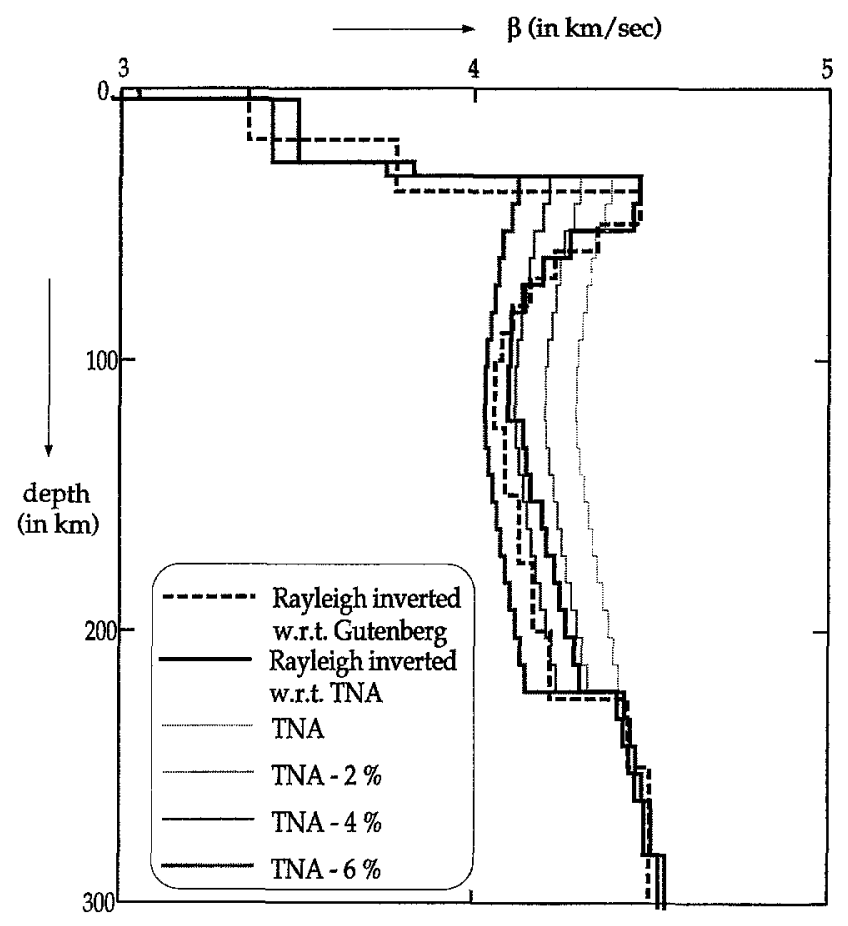

Figure 12. Shear-wave velocity-depth models. The gray lines show the TNA model and the models constructed by reducing $\beta$ by 2,4 , and $6 \%$ from the TNA model between crust and $225 \mathrm{~km}$. Also shown are the two inversion results: one with respect to $\mathrm{Gu}-$ tenberg (thick solid line) and the other with respect to TNA (thick dashed line).

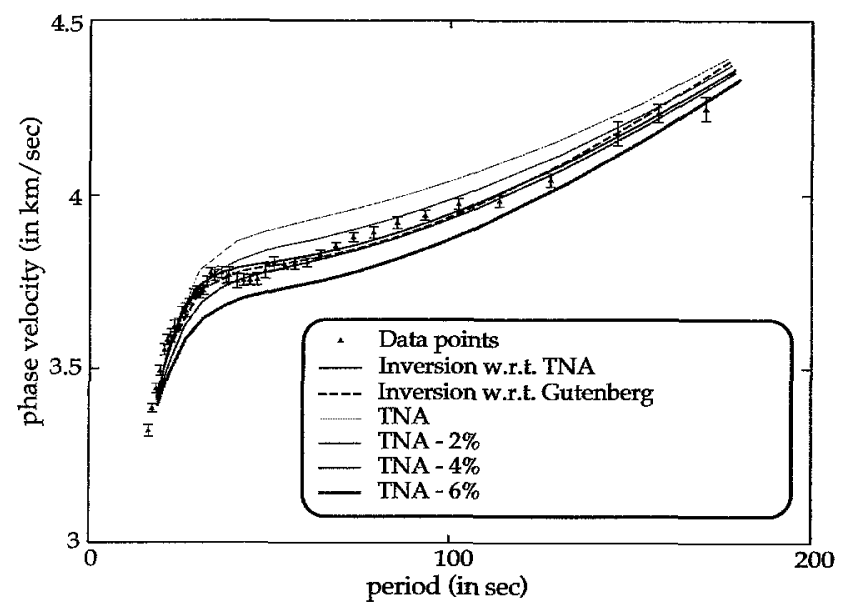

Figure 13. Dispersion curves for models shown in Figure 12, same annotation.

though the low velocity is less pronounced at greater depths. These two models obtained from two different starting models as well as the models derived from the TNA model strongly suggest that the shear $(S V)$ velocity in the upper mantle beneath southern California is on average 3 to $4 \%$ slower than the TNA model that represents western North America.
Also, the relatively high Love-wave velocity leads to an $S H / S V$ discrepancy of about $4 \%$. Note that we did not invert for $\eta$ or $P$-wave anisotropy. If we include $P$-wave anisotropy, the required $S$-wave anisotropy could be somewhat smaller. However, as mentioned earlier, since the $P$-wave partials are considerably smaller than the $S$-wave partial, the overall conclusion on the $S H / S V$ discrepancy would remain unchanged.

\section{Discussion and Conclusions}

The high-quality, very broadband TERRAscope data enabled us to perform accurate phase velocity measurements of Rayleigh and Love waves over a period range of 17 to $171 \mathrm{sec}$ and 49 to $146 \mathrm{sec}$, respectively. The results for different events agree well, warranting an inversion for the average velocity beneath southern California down to a depth of around $250 \mathrm{~km}$. It was not possible to explain Rayleighand Love-wave phase velocities simultaneously using a smoothly varying depth-velocity model. We performed a separate isotropic inversion to determine the $S H / S V$ velocity ratio that explains the observed Rayleigh/Love-wave incompatibility by a transverse isotropic model. The resulting velocity models ( $\beta_{R}$ for Rayleigh, $\beta_{L}$ for Love) show an anisotropy of about $10 \%$. However, synthetic seismograms, calculated using the $1066 \mathrm{~A}$ velocity model, show that at least part of this phase velocity incompatibility is due to contamination of the fundamental-mode Love wave with higher modes (the Rayleigh-wave phase velocities are not affected by higher-mode interference). We corrected for this highermode interference by subtracting the difference in phase velocities determined using fundamental-mode Love-wave synthetics, using velocity model $1066 \mathrm{~A}$, and using synthetics containing all modes, from the measured Love-wave dispersion. We found, however, that this correction cannot entirely account for the found Love/Rayleigh-wave discrepancy. We offer two possible mechanisms that, separately or in combination, can explain these observations: (1) There is anisotropy (around 4\% or less) present in the upper mantle in California. This anisotropy could be due to intrinsic anisotropy of olivine crystals in the upper mantle or a laminated structure with alternating high- and low-velocity layers (Toksöz and Anderson, 1963) that has not been properly modeled by body-wave studies. (2) The Rayleigh/Lovewave discrepancy can be accounted for in another way that does not require anisotropy. Possible mechanisms include contamination of Love-wave fundamental mode by higher modes. Even if our numerical experiment suggests that this effect is not large enough to explain the observed Love/ Rayleigh disparity, the proximity of the group velocities of the fundamental- and higher-mode Love waves could contaminate the fundamental mode more strongly than suggested by our numerical experiments using model 1066A. Another possible cause for the discrepancy is the uppermantle heterogeneity in southern California, such as the Transverse Range anomaly. It is possible that the heteroge- 
neity affects Love- and Rayleigh-wave velocities differently, so that it yields the apparent anisotropy. Numerical studies would be necessary to explore this possibility.

If the Love-wave data are not to be used, the $\beta_{R}$ velocity model represents the upper-mantle $S$ velocity structure beneath southern California. Our results for Rayleigh-wave dispersion show that the TNA model is not a representative model for the $S V$-structure beneath southern California. Velocities need to be 3 to $4 \%$ lower in the upper mantle to explain our measurements.

\section{Acknowledgments}

This research was supported by the USGS Grant Number 1434-95-G2554, 1934-HQ-97-GR-02989, and NSF Grant Number EAR 92-18809. Division of Geological and Planetary Sciences Contribution No. 5645.

\section{References}

Aki, K. (1961). Crustal structure in Japan from the phase velocity of Rayleigh waves, Bull. Earthquake Res. Inst. Tokyo Univ. 39, 255-283.

Aki, K. (1982). Three-dimensional seismic inhomogeneities in the lithosphere and asthenosphere: evidence for decoupling in the lithosphere and flow in the asthenosphere, Rev. Geophys. Space Phys. 20, 161170.

Anderson, D. L. and A. M. Dziewonski (1982). Upper mantle anisotropy: evidence from free oscillations, Geophys. J. R. Astr. Soc. 69, 383404.

Babuska, V. and M. Cara (1991). Seismic Anisotropy in the Earth, Kluwer Academic Publishers, Dordrecht, 217 pp.

Dorman, J., M. Ewing, and J. Oliver (1960). Study of shear-velocity distribution in the upper mantle by mantle Rayleigh waves, Bull. Seism. Soc. Am. 50, 87-115.

Ewing, M. and F. Press (1959). Determination of crustal structure from phase velocity of Rayleigh waves, part 3, the United States, Bull. Geol. Soc. Am. 70, 229-234.

Gilbert, F. and A. M. Dziewonski (1975). An application of normal mode theory to the retrieval of structural parameters and source mechanism from seismic spectra, Phil. Trans. R. Soc. London A, 278, 187-209.

Grand, S. P. and D. V. Helmberger (1984). Upper mantle shear structure of North America, Geophys. J. R. 76, 399-438.

Hadley, D. (1978). Geophysical investigations of the structure and tectonics of southern California. Thesis, California Institute of Technology, Pasadena, $167 \mathrm{pp}$.

Hadley, D. and H. Kanamori (1979). Regional S-wave variation for south- ern California from the analysis of teleseismic Rayleigh waves, $R$. Astron. Soc., Geophys. J. 58, 655-666.

Humphreys, E. D. and R. W. Clayton (1990). Tomographic image of the southern California mantle, J. Geophys. Res. 95, 19725-19746.

Jones, C. H. and H. Kanamori (1994). Missing roots and mantle "drips": Regional Pn and teleseismic arrival times in the southern Sierra Nevada and vicinity, California, J. Geophys. Res. 99, 4567-4601.

Kirkwood, S. C. (1978). The significance of isotropic inversion of anisotropic surface-wave dispersion, Geophys. J. R. Astr. Soc. 55, 131142.

Knopoff, L. (1972). Observation and inversion of surface wave dispersion, Tectonophysics 13, 497-519.

Knopoff, L. (1983). The thickness of the lithosphere from the dispersion of surface waves, Geophys. J. R. Astr. Soc. 74, 55-81.

McEvilly, T. V. (1964). Central U.S. crust-upper mantle structure from Love and Rayleigh wave phase velocity inversion, Bull. Seism. Soc. Am. 54, 1997-2015.

Mitchell, B. J. (1984). On the inversion of Love and Rayleigh wave dispersion and implications for Earth structure and anisotropy, Geophys. J. R. Astr. Soc. 76, 233-241.

Press, F. (1956). Determination of crustal structure from phase velocity of Rayleigh waves part I: southern California, Bull. Geol. Soc. Am. 67, 1647-1658.

Raikes, S. A. (1978). The temporal variation of tele-seismic P-residuals in southern California, Bull. Seism. Soc. Am. 68, 711-720.

Schlue, J. W. and L. Knopoff (1978). Inversion of surface-wave phase velocities for an anisotropic structure, Geophys. J. R. Astr. Soc. 54, 697-702.

Stange, S. and W. Friederich (1993). Surface wave dispersion and upper mantle structure beneath southern Germany from joint inversion of network recorded teleseismic events, Geophys. Res. Lett. 20, 23752378.

Takeuchi, H., J. Dorman, and M. Saito (1964). Partial derivatives of surface wave phase velocity with respect to physical parameter changes within the earth, J. Geophys. Res. 69, 3429-3441.

Thatcher, W. and J. N. Brune (1969). Higher mode interference and observed anomalous apparent Love wave phase velocities, J. Geophys. Res. 74, 6603-6611.

Toksöz, M. N. and D. L. Anderson (1963). Generalized two-dimensional model seismology with application to anisotropic earth models, $J$. Geophys. Res. 68, 1121-1130.

Wang, J. and T. L. Teng (1994). Surface-wave profiling of the lithosphere beneath the Mojave desert using TERRAscope data, J. Geophys. Res. 99, 743-750.

Seismological Laboratory

California Institute of Technology

Pasadena, California 91125

Manuscript received 29 January 1996. 